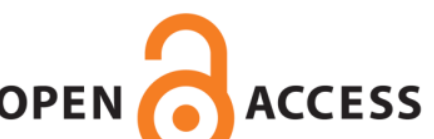

UWS Academic Portal

\title{
Achievable rate of hybrid precoding for hardware impaired MIMO underground mine channel
}

Belaoura, Widad; Ghanem, Khalida; Shakir, Muhammad Zeeshan; Nedil, Mourad; Bousbia, Hicham

Published in:

Electronics Letters

DOI:

10.1049/el.2018.8070

Published: 04/04/2019

Document Version

Peer reviewed version

Link to publication on the UWS Academic Portal

Citation for published version (APA):

Belaoura, W., Ghanem, K., Shakir, M. Z., Nedil, M., \& Bousbia, H. (2019). Achievable rate of hybrid precoding for hardware impaired MIMO underground mine channel. Electronics Letters, 55(7), 425-426.

https://doi.org/10.1049/el.2018.8070

\section{General rights}

Copyright and moral rights for the publications made accessible in the UWS Academic Portal are retained by the authors and/or other copyright owners and it is a condition of accessing publications that users recognise and abide by the legal requirements associated with these rights.

Take down policy

If you believe that this document breaches copyright please contact pure@uws.ac.uk providing details, and we will remove access to the work immediately and investigate your claim. 


\author{
On the achievable rate of hybrid precoding for hardware impaired MIMO underground \\ mine channel \\ Belaoura, Widad; Ghanem, Khalida; Shakir, Muhammad Zeeshan; Nedil, Mourad; Bousbia, \\ Hicham \\ Published in: \\ Electronics Letters
}

Accepted/In press: 06/02/2019

Document Version

Peer reviewed version

Link to publication on the UWS Academic Portal

Citation for published version (APA):

Belaoura, W., Ghanem, K., Shakir, M. Z., Nedil, M., \& Bousbia, H. (2019). On the achievable rate of hybrid precoding for hardware impaired MIMO underground mine channel. Electronics Letters.

\title{
General rights
}

Copyright and moral rights for the publications made accessible in the UWS Academic Portal are retained by the authors and/or other copyright owners and it is a condition of accessing publications that users recognise and abide by the legal requirements associated with these rights.

This is an Open Access item distributed under the terms of the Creative Commons Attribution-NonCommercial-NoDerivatives License (http://creativecommons.org/licenses/by-nc-nd/4.0/), which permits non-commercial re-use, distribution, and reproduction in any medium, provided the original work is properly cited, and is not altered, transformed, or built upon in any way.

Take down policy

If you believe that this document breaches copyright please contact us providing details, and we will remove access to the work immediately and investigate your claim. 


\section{On the achievable rate of hybrid precoding for hardware impaired MIMO underground mine channel}

W. Belaoura, K. Ghanem, MZ. Shakir, M. Nedil, and H. Bousbia-Salah

Hybrid precoding has become a well-accepted approach to enhance the achievable rate in the adverse millimeter-Wave (mm-Wave) multiple input multiple output (MIMO) channels. Transceiver impairments are inherent to any practical communication system, yet they are generally omitted when investigating the performance of wireless systems. More particularly, for the case of $\mathrm{mm}$-Wave underground channels, no study past been reported yet. To overcome this limitation, in this letter, a new To o architecture encompassing hybrid precoded mm-Wave MIMO structure, in which he transceiver processing, is presented. Furthermore, the geometrical one ring model is applied on the underground $\mathrm{mm}$-Wave measured channel on which the architecture is tested.

Introduction: Because of the future applications targeting underground mine environments, such as the wireless video, which are greedy in terms of data rate resources, different solutions offering such resources are sought. Owing to their large available bandwidth, millimeter-Wave (mm-Wave) communications are presenting themselves as a quite viable technology to fulfil such requirements, and the measurement campaigns to characterize such channels at this frequency band and adapt the communication to them, have already been launched [1]. One of the biggest impediments to $\mathrm{mm}$-Wave underground propagation is the presence of materials with different dielectric and conductive properties, of irregularities along the walls, as well as the high path loss, which hinders the communication scheme throughput enhancement [2]. To bridge the resulting significant link budget gap in such environments, directional multiple antennas have been recently adopted. Interestingly, scaling up the number of antennas, hence achieving a large spatial degree of freedom (DoF), yields a higher spectral efficiency (SE) and mitigates the effects of the channel fading $[3,4,5,6]$. However, most prior works on MIMO wireless systems neglect realistic aspects pertaining to the real deployment of the communication system, such as the imperfections of the transceiver hardware. Indeed, it is agreed upon that hardware components in a practical system experience various impairments, including the oscillator phase noise (PN) and the $60 \mathrm{GHz}$ power amplifier (PA) undesirable effects [7]. Motivated by this, this work proposes a hybrid precoding architecture, in which the previously mentioned hardware imperfections were included and compensated. The proposed solution is tested over the mm-Wave measured underground mine channel. Another contribution of this letter lies in the extension of the newly proposed 2-D geometrical scattering one ring-based model on measurements carried out in a mine in Northern Canada at the $60 \mathrm{GHz}$ band. To the best of our knowledge, neither the design of such a hardware impairments-aware scheme combining hybrid precoding-aided mmWave communications has been proposed before, nor the representation of underground channel with a 2-D geometrical scattering model has been targeted previously. The proposed architecture aims at enhancing the achievable rate performance in the adverse underground wireless $\mathrm{mm}$ Wave channels, while reducing the impingement of hardware transceivers practical defects on it. Our results corroborate that the architecture indeed meets such objectives in a viable way.

System model: Let us consider a hybrid precoding architecture deployed in a mm-Wave underground channel, wherein the transmitter and the receiver are equipped with $N_{t}$ and $N_{r}$ elements, respectively, as illustrated in Fig. 1. We assume that $N_{\mathrm{RF}}$ radio frequency (RF) chains are employed at the transceiver such that $N_{\mathrm{RF}} \leq N_{t}, N_{\mathrm{RF}} \leq N_{r}$, and that the total number of streams is $N_{s}=N_{\mathrm{RF}}$. In order to implement this architecture, the transmitter applies two consecutive precoding operations, $\mathbf{F}_{\mathrm{BB}} \in C^{N_{\mathrm{RF}} \times N_{s}}$, and $\mathbf{F}_{\mathrm{RF}} \in C^{N_{t} \times N_{\mathrm{RF}}}$, where the former refers to the digital baseband precoder, whilst the latter denotes the analog counterpart. The received signal can be then represented as

$$
\mathbf{y}=\mathbf{H F}_{\mathrm{T}} \mathbf{s}+\mathbf{n},
$$

where $\mathbf{s} \in C^{N_{s} \times 1}$ is the transmitted symbol, of which the covariance matrix is $\mathbf{Q}=\mathrm{E}\left[\mathbf{s s}^{H}\right]=\frac{P_{t}}{N_{s}} \mathbf{I}_{N_{s}}$, with $P_{t}$ being the total transmit power, $\mathbf{F}_{\mathrm{T}}=\mathbf{F}_{\mathrm{RF}} \mathbf{F}_{\mathrm{BB}}$ is the hybrid precoder, $\mathbf{H} \in C^{N_{r} \times N_{t}}$ is the underground mine channel at the $\mathrm{mm}$-Wave band emulated using the 2-D geometrical model, and $\mathbf{n} \sim C N\left(0, \sigma^{2}\right)$ represents the additive white Gaussian noise (AWGN) at the receiver, where $\sigma^{2}$ stands for the noise variance.

At the receiver side, the combiner $\mathbf{W}_{\mathrm{BB}} \mathbf{W}_{\mathrm{RF}}$, which is composed of an $\mathrm{RF}$ combiner $\mathbf{W}_{\mathrm{RF}} \in C^{N_{\mathrm{RF}} \times N_{s}}$ and a digital one $\mathbf{W}_{\mathrm{BB}} \in C^{N_{r} \times N_{\mathrm{RF}}}$, is used to extract the transmitted data from the received signal, which could be expressed as follows:

$$
\mathbf{y}=\mathbf{W}^{H} \mathbf{H F}_{\mathrm{T}} \mathbf{s}+\mathbf{W}^{H} \mathbf{n},
$$

This model implicitly assumes ideal transceiver hardware. To consider the impact of transceiver impairments which has been discussed in massive MIMO systems in [7], equation (2) can be modified as follows:

$$
\mathbf{y}=\mathbf{W}^{H} \mathbf{H}\left(\mathbf{F}_{\mathrm{T}} \mathbf{s}+\boldsymbol{\eta}_{t}\right)+\mathbf{W}^{H} \boldsymbol{\eta}_{r}+\mathbf{N},
$$

where $\mathbf{N}=\mathbf{W}^{H} \mathbf{n}$, and $\boldsymbol{\eta}_{t}, \boldsymbol{\eta}_{r}$ refer to the transceiver impairment residue in the transmitter and the receiver hardwares, respectively, which are assumed to be independent of the transmitted signal. These terms are modelled as

$$
\begin{gathered}
\boldsymbol{\eta}_{t} \sim C N\left(0, k_{t}^{2} \operatorname{diag}\left(\left|q_{1}\right|^{2}, \ldots,\left|q_{N_{t}}\right|^{2}\right)\right), \\
\boldsymbol{\eta}_{r} \sim C N\left(0, k_{r}^{2} \operatorname{tr}(\mathbf{Q}) \mathbf{I}_{N_{r}}\right),
\end{gathered}
$$

where the coefficients $k_{t}$ and $k_{r}$ are characterizing the levels of impairments at the transmitter and the receiver, respectively, with $q_{i}$ denoting the $i^{\text {th }}$ diagonal element of the signal covariance matrix $\mathbf{Q}$, and $\operatorname{tr}($.$) stands for the trace of the matrix$

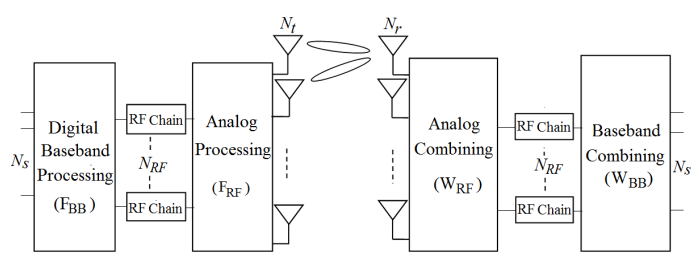

Fig. 1. Transceiver structure of hybrid mm-Wave MIMO system.

Geometrical-based Underground mine channel model: The geometrical one ring model for MIMO underground mine channel is shown in Fig. 2, in which the transmitter is equipped with $N_{t}$ antennas and the receiver with $N_{r}$ antennas. It assumes that there are $L$ scatterers $S_{l}(l=1, \ldots, L)$ located on a ring, with a radius $\mathrm{R}$ around the receiver. The measurements carried out in a real underground mine operated by the Canadian Center for Minerals and Energy Technology (CANMET) are exploited in our model. During the experiments, the transmitter (Tx) is maintained fixed, while the receiver $(\mathrm{Rx})$ was moved at different positions along the gallery, from $1 \mathrm{~m}$ up to $10 \mathrm{~m}$, with steps of $1 \mathrm{~m}[1]$

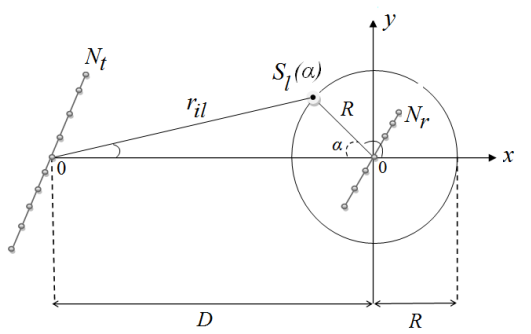

Fig. 2 Geometrical one ring model for a MIMO Underground mine channel [8].

In the one-ring model the path length from the $i^{t h}$ transmit antenna to the $r^{\text {th }}$ receive antenna via the $l^{\text {th }}$ scatterer is obtained through geometrical considerations as a function of $D$ :

\section{ELECTRONICS LETTERS Vol.00 No.00}




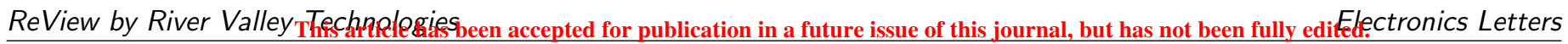
Content may change prior to final publication in an issue of the journal. To cite the paper please use the doi provided on the Digital Library page.

$$
d_{i l r}=R+\sqrt{\left(D^{2}+R^{2}-2 D R \cos (\alpha)\right)}
$$

where $\alpha=\arcsin \left(\frac{r_{i l}}{D}\right)$ is the angle of arrival of the $l^{\text {th }}$ scatterer, and $D$ is the distance between the Tx and Rx. Thus, the corresponding channel in the $r^{t h}-i^{t h}$ link is given by:

$$
H_{i, r}=\frac{1}{\sqrt{L}} \sum_{l=1}^{L} \sqrt{B_{l i, r}} e^{\left(-j 2 \pi\left(\frac{d_{i l r}}{\lambda}\right)+j \phi_{l i, r}\right)}
$$

where $B_{l i, r}$ is the $l^{\text {th }}$ measured time domain path loss obtained from the channel impulse response, for any fixed location between Tx and
$\operatorname{Rx}, \phi_{l i, r}$ is the phase shift associated with each scatter $S(\alpha)$, which is assumed in our case as uniformly distributed in the interval $[-\pi, \pi]$.

Simulation Results: In this section, the achievable rate performance is investigated for the proposed system incorporating hybrid precoding technique, over the MIMO underground mine channel, for which measurements have been fitted using geometrical model. Analog beamforming, implemented within a similar architecture as the proposed scheme, and relying on the one-ring channel model, is taken as a reference to study the performance penalty with hardware defaults. The system considers the practical hardware imperfections at the both transmitter and receiver sides, and is operating in the frequencies ranging from 57 to $62 \mathrm{GHz}$, thus has a bandwidth of $500 \mathrm{MHz}$. Furthermore, it is assumed that the transmitter and the receiver are equipped with equal number of antenna elements, the number of RF chains $N_{\mathrm{RF}}=2$ and the number of scatterers in the geometrical model has been assumed as $L=3$. Based on this, the normalized achievable rate $R$ of hybrid precoding scheme incorporating transceiver hardware impairments could be formulated as:

$$
R=\log _{2} \operatorname{det}\left(\mathbf{I}_{\mathrm{Ns}}+\frac{\gamma}{N_{\mathrm{Nt}}}\left|\mathbf{W}^{H} \mathbf{H F}_{\mathrm{T}}\right|^{2} \boldsymbol{\Phi}^{-1}\right)
$$

where $\mathbf{\Phi}=\gamma k_{t}^{2} \mathbf{F}_{\mathrm{T}} \mathbf{F}_{\mathrm{T}}^{H}+\left(\gamma k_{r}^{2}+1\right) \mathbf{I}_{\mathrm{Nr}}$ represents the hardware impairments, and $\gamma$ is the signal to noise ratio (SNR).

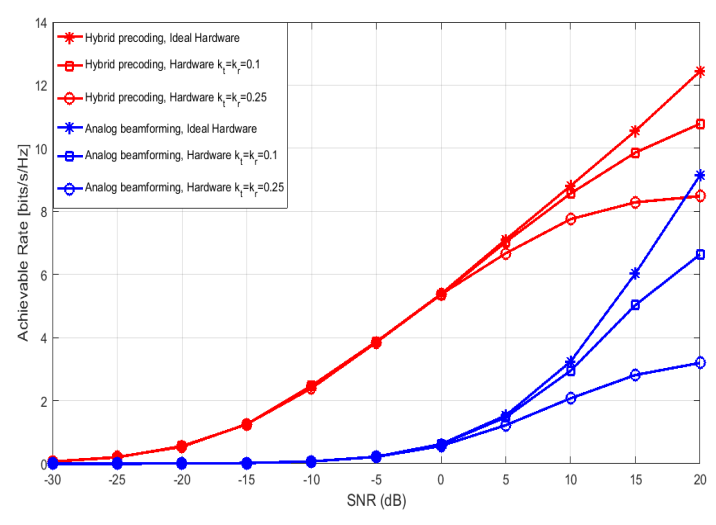

Fig. 3 Performance comparison of the proposed system in the modeled underground mine channel with different levels of impairments.

The simulation investigates the result of increasing the effect of hardware impairments on the proposed system. For this purpose, the parameters $k_{t}$ and $k_{r}$ are varied to emulate the variation of the degree of impairments from high to non-existent, and, without loss of generality, the numbers of transmit and receive antennas are both equal to 10 From Fig. 3 comparing the behaviour of the proposed scheme along with analog beamforming alternative face to hardware defaults, it is seen that a performance loss is noted relative to the idealistic hardware operation conditions, more particularly with analog beamforming, and increases substantially as the impairment level gets higher. The presence of these practical imperfections induces a saturation phenomena in the achieved rate at relatively high SNR values, where the increase of the transmit power brings no gain in the performance. It is also observed that analog beamforming-based system experiences a critical performance penalty which exceeds $6 \mathrm{~dB}$, when the hardware conditions vary from being ideal to undergoing an impairment factor of 0.25 at both the transmitter and the receiver sides. By contrast, the proposed solution exhibits a significant robustness to such hardware deviations from ideal case, and the corresponding noted performance losses remain quite reasonable. The presented architecture outperforms analog beamformingbased alternative regardless of the conditions of operations of the hardware, and is then more appropriate for practical deployments in such underground channels.

Conclusion: This letter considers the hardware distortions, pertaining to the transmit and receive RF chains, in the design of the hybrid precoded $\mathrm{mm}$-Wave MIMO architecture, deployed in underground channels. We have applied a 2-D geometrical channel model, which is based on one ring configuration, to describe the propagation mechanism in our underground channel, and achievable rate performance was investigated based on this model and the modified transceiver structure. The higher performance and robustness against hardware imperfections of the proposed solution, relative to analog beamforming-based counterpart, have been demonstrated in these MIMO underground environments.

W. Belaoura, and H. Bousbia-Salah (Electrical Engineering Department, Ecole Nationale Polytechnique, Algiers, Algeria)

E-mail:kghanem@cdta.dz

K. Ghanem (Telecommunications Laboratory, CDTA Algiers, Algeria)

MZ. Shakir (School of Engineering and Computing, UWS, Paisley, Scotland, UK)

M. Nedil (Underground Communications Research Laboratory LRTCS, UQAT, Canada)

\section{References}

1 Ghaddar, Mohamad and Talbi, Larbi and Nedil, Mourad and Mabrouk, Ismail Ben and Denidni, Tayeb A.: 'Mm-waves propagation measurements in underground mine using directional MIMO antennas', IET Microwaves, Antennas \& Propagation, 2016, 10, (5), p. 517-524

2 El Azhari, M and Nedil, M and Alj, YS and Mabrouk, IB and Ghanem, $\mathrm{K}$ and Talbi, L: 'Off-body LOS and NLOS channel characterization in a mine environment', Electrical and Information Technologies (ICEIT) 2015 International Conference on, 2015, pp. 114-118

3 Alkhateeb, Ahmed and El Ayach, Omar and Leus, Geert and Heath, Robert W.: 'Channel estimation and hybrid precoding for millimeter wave cellular W.: 'Channel estimation and hybrid precoding for millimeter wave cellular
systems', IEEE Journal of Selected Topics in Signal Processing, 2014, 8 , systems', IEEE Jour
(5), pp. 831-846

4 Ghanem, K.: Effect of channel correlation and path loss on average channel capacity of body-to-body systems', IEEE transactions on Antennas and propagation, 2013, 61, (12), pp. 6260-6265

5 Rusek, Fredrik and Persson, Daniel and Lau, Buon Kiong and Larsson, Erik G and Marzetta, Thomas L and Edfors, Ove and Tufvesson, Fredrik. 'Scaling up MIMO: Opportunities and challenges with very large arrays', IEEE Signal Processing Magazine, 2013, 30, (1), pp. 40-60

6 Belaoura, Widad and Ghanem, Khalida and Bousbia-Salah, Hicham. 'Hybrid precoding for DL massive MU-MIMO systems with distributed antenna deployments', Antennas and Propagation \& USNC/URSI National Radio Science Meeting, 2017 IEEE International Symposium on, 2017, p. 1173-1174

7 Björnson, Emil and Matthaiou, Michail and Debbah, Mérouane.: 'Massive MIMO with non-ideal arbitrary arrays: Hardware scaling laws and circuitaware design', IEEE Transactions on Wireless Communications, 2015, 14, (8), pp. 4353-4368

8 Shiu, Da-Shan and Foschini, Gerard $\mathrm{J}$ and Gans, Michael $\mathrm{J}$ and Kahn, Joseph M.: 'Fading correlation and its effect on the capacity of multielement antenna systems', IEEE Transactions on communications, 2000, 48, (3), pp. 502-513 